Archives de sciences sociales des religions

110 | avril-juin 2000

Varia

\title{
De la quête de Dieu à la question de la femme : les états de mystique
}

Daniel Vidal

\section{(2) OpenEdition}

1 Journals

Édition électronique

URL : http://journals.openedition.org/assr/20202

DOI : $10.4000 /$ assr.20202

ISSN : 1777-5825

Éditeur

Éditions de l'EHESS

Édition imprimée

Date de publication : 1 juillet 2000

Pagination : 41-51

ISBN : 2-222-96691-4

ISSN : 0335-5985

Référence électronique

Daniel Vidal, «De la quête de Dieu à la question de la femme : les états de mystique », Archives de sciences sociales des religions [En ligne], 110 | avril-juin 2000, mis en ligne le 19 août 2009, consulté le 01 mai 2019. URL : http://journals.openedition.org/assr/20202 ; DOI : 10.4000/assr.20202

Ce document a été généré automatiquement le 1 mai 2019

(C) Archives de sciences sociales des religions 


\title{
De la quête de Dieu à la question de la femme : les états de mystique
}

\author{
Daniel Vidal
}

\section{RÉFÉRENCE}

MAîTRE (Jacques), Anorexies religieuses, anorexie mentale. Essai de psychanalyse sociohistorique. De Marie de l'Incarnation à Simone Weil, Paris, Cerf, 2000, 197 p. BRUNEAU (Marie-Florine), Women Mystics Confront the Modern World - Marie de l'Incarnation (1599-1672) and Madame Guyon (1648-1717), Albany, State University of New York Press, 1998, $279 \mathrm{p}$. BERNARD (Charles-André), Le Dieu des mystiques, Tome II : La conformation au Christ, Paris, Cerf, 1998, $734 \mathrm{p}$. KRUMENACKER (Yves), L'École française de spiritualité. Des mystiques, des fondateurs, des courants et leurs interprètes, Paris, Cerf, 1998, 660 p.

1 De l'ouvrage de Jacques Maître sur les anorexies religieuses, et de l'interrogation par Yves Krumenacker du bien-fondé d'une école française de spiritualité, à l'analyse, par CA. Bernard, du «Dieu des mystiques», et à l'affirmation de modernité de la mystique féminine par M.-F. Bruneau, - une nouvelle floraison de textes majeurs vient occuper la scène de la sociologie et de l'histoire de la spiritualité européenne. Au centre de cette passion d'écritures, et en contrepoint de la disparité des thèmes et interprétations, deux questions que chaque auteur pose avec précision et en urgence de réponse : qu'en est-il de la femme dans la vocation à Dieu; qu'en est-il de Dieu dans l'intimité de l'univers féminin? C'est autour de cette raison de connaissance que s'organisent les livres présentés ici.

2 Ainsi du dernier ouvrage de Jacques Maître, concernant les états du corps féminin, en tant qu'ils réalisent en l'expérience mystique les catégories de la subjectivité.

3 En quelques années décisives, J. Maître a livré aux sociologues des phénomènes religieux, et plus largement aux chercheurs en sciences sociales, une véritable somme sur différents 
aspects de la mystique affective féminine dans l'Occident catholique ${ }^{1}$. Sa "tétralogie ", qui fut présentée ici en détail, ne permettait pas seulement de faire rencontre avec des figures ayant fait irruption dans l'institution du religieux sans déborder ce cadre même, et ses meilleurs connaisseurs. Elle autorisait un regard nouveau sur ces grandes aventures de l'esprit que sont les expériences mystiques. Ce regard procède d'une méthode liant en une seule instance d'intelligibilité la connaissance approfondie de l'itinéraire mystique chaque fois singulier du fidèle entrant en passion de son dieu, l'inscription de cette expérience de spiritualité à même les instances et conflits sociaux dont elle est l'extrême contemporaine, et l'argument analytique, qui fonde toute épreuve, et celle-ci de façon exemplaire, sur les tourments, et tournants, de la subjectivité. Nulle juxtaposition de méthodes de lecture disparates, qui viendraient à convergence par la vertu d'un pari épistémologique. Mais une mise en intrigue rigoureuse, qui formule, dans les limites du champ ainsi défini, les hypothèses les plus aptes à rendre compte, dans l'universalité des enjeux mystiques, la singularité de chaque « style».

4 Cette méthode qui tresse l'« écoute » analytique à la compétence sociohistorique, guide aujourd'hui l'A. dans l'exploration d'une modalité d'action qui, dans les théologies bien tempérées, au moins à partir du XVII ${ }^{e}$ siècle, se heurta aux plus fortes réserves de la part des instances religieuses. Les conduites anorectiques, qui brisaient le corps et le réduisaient à n'être que témoignage d'une passion de type christique, ne peuvent plus s'entendre selon ces seuls critères: le corps en inanition n'exhibe pas sa seule nervure d'abjection et de mortification. J. Maître insiste avec force sur la pluralité de ces « langages du corps » dans la spiritualité féminine, la « diversité de leur réalisation ». Ils ne parlent pas d'un corps féminin venu et reclus en sa pauvreté essentielle, mais constituent des « façons anorectiques d'être au monde ». D'emblée, l'A. propose une grille de lecture où l'ensemble des considérants analytiques et historiques vient authentifier l'anorexie non comme offrande sacrificielle, mais comme irruption d'une subjectivité insoumise à l'ordre du monde, récapitulant, en ce scandale, quelque trauma impossiblement assumé, parce que toujours en frontière d'oubli et de dénégation, cependant au foyer d'une mémoire vigilante.

5 L'anorexie apparaît alors l'une des solutions les plus radicales qu'une femme puisse inventer en mystique pour demeurer contemporaine de son destin personnel, métissé de négation de soi et, sans doute de façon centrale, de «relation problématique » avec la mère, ou son tenant lieu. Mais ce choix anorectique, s'éprouvant dans le cadre d'une quête personnelle mobilisant le principe d'altérité - Dieu comme tout autre -, se dégage aussitôt de toute qualification pathologique. Il n'est pas symptôme de dérive psychique, moins encore théâtralisation d'une crise intime. Il atteste d'un "destin pulsionnel » au plan subjectif, dans le temps même où il se déploie dans l'« adhésion à une discipline religieuse instituée ». J. Maître ne s'en tient pas seulement à cette mise au net : si en toute anorexie il convient de nommer la pulsion de mort à l'œuvre, il est aussi nécessaire de distinguer, dans la longue théorie des anorexies religieuses, celles qui obéissent d'emblée à des schèmes institutionnels qui, pour ainsi dire, les sollicitent en tout leur "style »anorexies «canonisées", ou "saintes»-, et celles, qui l'occupent ici, anorexies «mystiques» par excellence. L'A. définit leur raison nucléaire: refus d'assumer la maternité, refus d'entrer comme objet dans un procès d'échange et de transaction économique. Où l'on voit qu'en effet l'écoute clinicienne et la mise en regard de l'inscription sociale fondent le lieu d'excellence de l'interprétation. 
6 Les anorexies mystiques sont ainsi marques d'insoumission paroxystique : par elles la désaffiliation s'accomplit jusqu'à son terme; par elles aussi l'acte marchand s'annule asymptotiquement. De précieux paradoxes s'en suivent. La récusation de la féminité du sujet s'effectue par le recours à la féminisation des figures de la foi : J. Maître en avait en un autre ouvrage longuement explicité la raison - féminité de Jésus, exaltation de la Vierge, emblématique de la lactation, jouissance et extase dans l'écoulement en son dieu et de Dieu en elle, etc. Plus la mystique féminine opère à la subversion des "fonctions » sociales et biologiques de la femme, plus elle participe de l'expansion quasi illimitée de l'univers du féminin. Pourquoi n'irait-on pas jusqu'à dire que cette subversion fonde, selon sa logique propre, quelque triomphe de ce sujet en tant que femme. De la même façon, soustraite au principe de négociation, la femme œuvre en mystique à la sortie du monde régi par les règles de l'échange. Mais, parvenue en effet à n'être plus que ce « rien » à quoi aspire toute spiritualité, elle se trouve du même coup en quelque absolu sans doute, mais terme également d'un interminable échange, puisque équivalente à toute autre.

7 L'« inédie », ce « jeûne absolu », est effet de récit plus que pratique réelle. Du moins peuton, par ce récit même, par ce mythe, identifier les pôles autour desquels s'organise le corps anorectique, et là encore de nouveaux paradoxes se développent : ce corps que l'on veut éteindre, on le veut aussi incorruptible, perpétuant donc sous les espèces de l'éternité, une présence que l'on récuse pour le temps que l'on habite. Du corps épuré au corps "sacramentalisé ", on suit l'itinéraire d'une passion mortifère, qui exhibe d'autant plus ce corps qu'elle veut en finir avec lui. Des grandes anorectiques mystiques, dont l'A. présente en la première partie de son ouvrage quelques figures remarquables, investissent le plus profond, et le plus trouble, de leur subjectivité, dans un cheminement religieux qui les soustrait à toute qualification pathologique originelle. J. Maître propose ici une hypothèse que je pense très novatrice. Loin que la mystique ait été, au long des siècles où elle ouvrit une béance majeure dans la théologie, la voie par quoi un affolement de raison pouvait se dire, il se pourrait que ce que l'on nomme aujourd'hui anorexie mentale soit l'ultime résultat d'un procès de désenchantement et de laïcisation d'un sujet privé de miroir. Et qui n'existe plus, précisément, que comme par principe hors de soi. Quand la mystique ne se concevait que comme opération de stricte intériorité, l'anorexie œuvrait à même celle-ci, et lui conférait sa capacité à bouleverser l'ordre intime du monde. Dès lors que ce «recueillement intérieur", pour reprendre les catégories des mystiques du Grand Siècle, ou cet " espace privé », pour parler la langue d'aujourd'hui, semblent de moins en moins autorisés à se vivre pleinement, - seule une qualification médicale serait capable de rendre compte de ces déficits de l'esprit en les nommant tels, quand ils attestaient au contraire une expérience singulière quant au corps et à ses paroles. Étrange retournement épistémologique: dans l'argumentaire conduisant à la médicalisation de l'anorexie "mentale ", il conviendrait, le traquant dans ses censures et ses trompe-l'œil, de continuer à déchiffrer ce qui s'y livre de verbes essentiels à une exacte vision du réel. Sous le "simulacre» de la maladie mentale, une expérience spirituelle serait-elle toujours en cours?

8 Les deux figures majeures que J. Maître présente dans la deuxième partie de son ouvrage - Marie de l'Incarnation au XVII ${ }^{\mathrm{e}}$ siècle, Simone Weil au XX $\mathrm{X}^{\mathrm{e}}$ - autorisent à poser la question. Deux cas d'anorexie assez dissemblables: Marie développe un comportement anorectique d'intensité relativement faible - plus « maltraitance de soi-même » que mise en perte de son corps. Simone, au contraire, mourra d'inanition, son corps consumé. 
Toutes deux présentes à leur siècle et leur histoire comme il en va des "virtuoses » en mystique - Thérèse d'Avila serait ici visage emblématique. Présence de telle intensité, de tel acharnement, qu'on ferait contresens de l'opposer à ces modes anorectiques « d'être au monde ». Ce monde en est au contraire l'issue et la condition. L'anorexie de Marie porte refus sans pitié de la maternité, du maternage, et du fils, et œuvre au plein du siècle et des missions que l'Église entreprend alors. Simone raréfie sans cesse son «besoin de corps ", prend "résolution d'être homme le plus possible ", lamine sa chair et s'installe dans une anorexie sans fin, - et dans le même temps connaît la misère ouvrière, participe aux luttes sociales, et aux idéologies les plus radicales: communisme oppositionnel, syndicalisme révolutionnaire, etc. J. Maitre se dispose au cœur de cette intrigue passionnante, qui conjoint l'acte le plus décisif de mise en abstraction du corps hors de l'orbe du monde, et la passion de ce monde même, au risque de s'y perdre. Si l'on accepte l'argument de l'A., à coup sûr la plus engagée en mystique par sa décision même d'anorexie pourrait bien n'être pas Marie, la femme à qui l'on est convié de penser, mais bien Simone Weil. Ses énoncés participent de cette vaste diction qui traverse les siècles, et connaît avec elle une façon d'exact accomplissement. Aussi bien ai-je été conduit à ne pas partager sur ce point la conclusion de l'A., qui tend à privilégier malgré tout pour notre contemporaine le diagnostic d'anorexie mentale, tous les critères évoqués demeurant «chez elle hors du cadre tracé par la sacralisation ecclésiale de l'anorexie mystique ». Ce serait, à terme, constituer l'Église seule gestionnaire des " biens de salut », quand d'autres instances aujourd'hui - espace public, éthique, morale civique, qui émergent du procès même de désenchantement - définissent d'autres enjeux que de salut personnel, en d'autres lieux de légitimité que l'institution ecclésiale. Mais ce n'est là que remarque incidente. Elle ne veut que confirmer la richesse d'une méthode croisant des univers référentiels auparavant disjoints, et la fécondité d'un théorème épistémologique, que cet ouvrage ainsi consacre.

De Marie de l'Incarnation, l'ouvrage de Marie-Florine Bruneau traite à son tour, et de Madame Guyon.

La mystique féminine n'en finit pas de susciter l'intérêt des praticiens des sciences sociales. Comme si, porteuse d'un défi majeur dans l'ordre de la connaissance des faits de religion, elle récusait toute lecture réductrice à quelque excès d'affectivité, ou à quelque affluence intempestive du corps en ses désirs et son identité sexuée. Les travaux de J.N. Vuarnet ou de J. Maître, entre autres, ont croisé différents registres d'interprétation, pour tenter précisément de dégager la spécificité, en même temps que l'universalité, de cette expérience mystique. L'ouvrage de M.-F. Bruneau relève à son pour de cette exigence de lecture compréhensive, aux frontières de la psychologie, de l'épistémologie, et de ce qu'on pourrait appeler une phénoménologie historique des genres.

11 L'A. s'inscrit dans une double référence. À M. de Certeau, qui proposa une interprétation de la mystique à partir des concepts de la psychanalyse et de la sémiologie, et rendit compte de la progressive érosion de la spiritualité au XVII e siècle, face à l'émergence de la science moderne - les femmes en mystique, mais plus généralement l'énoncé mystique lui-même, marqués alors d'« extravagance » et se marginalisant au sein de l'institution, $a$ fortiori de la société de leur temps. À Caroline W. Bynum, qui, dans ses travaux pionniers sur les mystiques féminines du haut Moyen Âge ${ }^{2}$ institue la femme en mystique comme proposant une nouvelle perception du corps et de sa subjectivité. L'A. assume cette double référence, mais selon un argumentaire qui l'autorise à en dépasser les limites propres. 
12 C'est au fond la chance des deux mystiques présentées dans l'ouvrage, que de se situer en des discontinuités d'histoire. Marie de l'Incarnation et Mme Guyon, à une génération d'intervalle, vivent les dernières flambées d'une spiritualité venue du fond des siècles rhénans, et qui les habite passionnément, et les premières heures d'un monde qui s'ouvre au savoir scientifique. Mais ces deux temporalités se renforcent, paradoxalement, plus qu'elles ne se combattent. Au moins sur un plan, qui concerne précisément la question de la femme. Et la signification de la surrection de son corps, sa rage d'écriture, sa capacité de "résistance » aux interdits et stigmatisations dont elle est alors victime, et qui désormais la disposent à perte de mémoire. Contre les canons établis, à l'époque de la grande effusion mystique, dont les femmes furent sujets d'excellence, corps, visions, ravissements, extases, et tout ce qui se trame de signes singuliers dans le travail de Dieu, constituent non seulement une défense de la femme comme actrice majeure de la spiritualité, mais surtout comme habilitée à dire son corps sexué haut lieu de stratégie quotidienne de lutte contre les rationalités propres à chacun de ces temps. La même leçon vaut face à la modernité scientifique : aux définitions instituantes de la "féminité », ces femmes se soustraient par excès : surcharge d'écriture chez Mme Guyon, et déploiement d'un espace infini de commentaires du Livre ; chez Marie de l'Incarnation, surabondance de grâces dès lors qu'elle aborde le Nouveau Monde, et germination impatiente de subjectivité propre, une fois récusé en grande violence le statut de mère.

13 Mais il y a plus. Le corps féminin est en mystique porté à ses frontières de sens. En imitation du corps christique, il est à la fois raison de souffrance et lieu même de rédemption, en ses défaillances et ses outrances. Et dans le temps où elles participent de cette conformation à la Passion, à son coefficient historique, à la présence dont il témoigne, Marie et Jeanne attestent la convenance de leur œuvre avec l'héritage de la théologie négative. Peut-être cette hypothèse mériterait-elle d'être examinée plus à fond. Mais il est vrai que l'une et l'autre puisent le plus profond de leurs inspirations, et l'occasion de leur plus grand scandale du corps et d'âme, dans les réseaux de spirituels formés à l'école de la mystique abstraite, autour de Benoît de Canfield et de la Perle Évangélique, cet ouvrage venu du fond des béguinages flamands 3 .

14 La mystique féminine se trouve ainsi aux prises avec une double injonction. L'instance du corps qu'elle déploie en toutes ses failles et insolences, n'est pas ultime refuge d'une identité de femme niée. Ou pas seulement. Ce corps est conquérant: en lui, en son offrande forcément sexuée, se formule, s'invente et recommence à chaque étape de la quête mystique, la femme comme sujet emblématique. Non pas sujet particulier, qui réclame qu'on prenne en compte sa valeur propre à partir de son statut féminin. Mais, portée à la plénitude de sa formule, la femme comme passage obligé pour toute institution du sujet. Car ces femmes en mystique, d'inamovible soumission, ont fondé, par l'irruption de leur corps, et fût-ce par recours à des actes et signes de plus grande ombre, l'espace d'une «liberté intérieure ». L'A. marque avec force qu'ainsi Marie non plus que Jeanne ne subvertissent, à rigoureusement parler, l'ordre social et culturel dont elles relèvent, mais acquièrent infiniment plus : " pouvoir et transcendance » - capacité à dire, avec Angèle de Foligno, et la rencontre avec son dieu, et la naissance de la femme comme absolu de tout sujet.

De là un autre impératif. Par le bouleversement que la femme installe dans la cité, il s'ensuit que la mystique est arrachement continu au monde tel qu'il va. Et de telle ablation, qu'il se pourrait qu'en ce procès de mise en perte, elle-même s'y s'abolisse. L'écriture de Marie de l'Incarnation, celle de Jeanne Guyon, ne sont pas simples 
témoignages d'une expérience d'exception, ou interprétations allégoriques. Elles opèrent en même temps à la dissolution du lieu même d'où elles parlent. Marie écrit, et c'est acte, dit l'A., de « distanciation", soumis à " double contrainte ». Il faut que le sujet identifié par son marquage historique, se rende «invisible» comme narrateur de sa propre histoire - celle-ci dès lors abstraite et comme sans mémoire; mais il faut aussi bien que cette écriture soit pure "représentation de soi », parole de for intérieur, attestation d'intériorité comme espace d'assez large emprise pour englober le monde du dehors. Jeanne écrit, en telle urgence qu'on la dit « graphomane ». Mais cette écriture ne se réduit pas à la véhémence de son contenu, et aux apories de sa lettre. Elle est tout entière exhibition de féminité: aussi bien offusquera-t-elle Bossuet et Fénelon. Soit son autobiographie: métissée d'histoire, d'innovation littéraire, et de prise en compte des bouleversements épistémologiques alors en travail dans le siècle, elle annonce certes Rousseau. Mais par le brouillage du sujet de cette écriture, Mme Guyon fait imploser le système traditionnel de référence : elle est « auteur » de la narration, elle en est l'objet, elle n'écrit que sous l'inspiration de Dieu. Certes, cela est de commun partage avec d'autres "récits", notamment médiévaux. Mais dans le cadre de l'affirmation d'une féminité nouvelle, il convenait qu'en cette fin du XVII e siècle, se résorbe peu à peu la mystique et sa logique discursive, pour que corps de l'écriture, corps de femme, et nouvel horizon de la modernité scientifique ne soient qu'un. La mystique participe ainsi à la mise en place d'un nouveau champ épistémologique, fondé sur une conception neuve du sujet, dont on sait désormais qu'elle est aussitôt à raison de féminité.

L'irruption de la féminité dans la raison mystique constitue l'une des topiques fondatrices de la qualification de Dieu telle que formulée par corps et âme en la spiritualité occidentale. En témoigne l'ouvrage de Charles-André Bernard.

17 Est-il un dieu des mystiques qui ne serait pas de commun partage avec le dieu de l'anonyme fidèle ? À moins que celui-ci n'entre en sa connaissance, ou son regard, sans en avoir science même? Cette dernière hypothèse sera soutenue tout au long des siècles de spiritualité occidentale, par quelques-uns des plus illustres penseurs de la mystique, qui disent la rencontre insoupçonnée entre la plus radicale ignorance des fins, et ces fins elles-mêmes. À l'évidence, ce n'est point ici la thèse de C.-A. Bernard, et cela nous vaut cet ouvrage d'une rare densité, interrogation sur les voies de la mystique, les modalités de la quête, ses instances et ses tournures. En un livre précédent ${ }^{4}$, l'A. s'était attaché à l'étude de la relation dynamique "entre sujet spirituel et le terme auquel il tend». Son questionnement demeurait explicitement circonscrit à "l'espace intérieur", où s'accomplissent des interlocutions intimes, des mises à l'épreuve de l'esprit, et des réseaux de réciprocité entre la créature et son dieu, fondés sur les différentes "puissances» et facultés de l'âme, et leur combinatoire. À cette mise au net de cette « expérience » intime, singulière, il convenait que réponde l'examen minutieux de cette autre « expérience » à la frontière du corpus doctrinal, de l'assertion théologique et de la spiritualité, et qui concerne le recours à la figure médiatrice du Christ.

18 Tel est donc l'enjeu de cet ouvrage : pour que s'opère l'« union mystique », indiquer les modes explicites de "participation au mystère " de la croix, chacun de ces modes obéissant à l'impératif de "conformation » au Christ. Pour cette recherche au principe d'une des «voies» mystiques les plus paradoxales, l'A. mobilise des compétences interprétatives et des ressources de savoirs exceptionnelles. Voie paradoxale : au fur et à mesure que, s'écartant du paradigme de la pure intériorité, l'ouvrage informe sur les différents modes de "perception expérientielle» dénotant les énoncés mystiques, on 
observe quelque chose comme un éclatement de la spiritualité, partagée entre une perspective proprement religieuse, qui va se réaliser en théologie renouvelée, notamment avec Bérulle et dans son prolongement, et une formule mystique qui, mettant au centre de son énoncé la question du sujet, revient obliquement à sa source, cet espace intérieur où tout se consommait alors.

La raison du Christ est ici première : plus, peut-être, que son imitation, - le devoir de conformité. De saint Bernard à Bérulle, de François d'Assise à Thérèse de Jésus, C.-A. Bernard interroge les façons de cette économie sacrificielle, oblative, d'amour et de désespérance. Des grandes figures des premiers temps chrétiens, Ignace d'Antioche, Polycarpe, à Jean de Brébeuf, la « communauté de destin » s'éprouve à la lettre : voici le martyre, ce témoignage ultime, cette expérience exemplaire. Bien sûr, ceci est l'exception : mais la règle est posée dès l'origine - il n'est de voie vers son dieu que par la médiation du fils. En tant que venue historique. «Incarnation », « Verbe incarné », disent les mystiques au travers des siècles. Cette règle consentie, ses accomplissements sont multiples. C'est là que l'A. déploie des trésors d'analyse toute de finesse et de rigueur. Pour répondre à cette question : « Quels sont les profils objectifs que présente la mystique chrétienne au cours de son développement?» (pp. 99-100). On peut sans doute discuter la pertinence de l'opposition entre les références à l'«anatomie de l'âme», qui renverraient, chez Thérèse d'Avila et Jean de la Croix, à une théorie des instances mentales à l'œuvre dans l'oraison mystique, - et la phénoménologie de la « participation concrète au mystère de la foi ». Mais en ces deux grands " profils », une leçon commune : la pauvreté comme "valeur en soi ", l'amour comme "abaissement ». Est-on dès lors si éloigné de la thématique de l'intériorité, où se combinent en effet ces constellations ? fûtce en d'autres termes, à l'évidence: néant, nudité, désappropriation, etc. Au reste, il suffit, semble-t-il que des femmes entrent en mystique, pour que perception affective de l'union à Dieu, et son cortège de visions et révélations, s'articulent à nouveau à l'impératif essentialiste de néantisation. Qui est au principe de l'exaltation de la créature comme sujet, à quoi aboutissait la spiritualité flamande. Soit Angèle de Foligno, excessivement femme, proie, dit l'A., de "radicalité évangélique ». Femme debout dans la " très grande illusion », faisant « expérience unique de la ténèbre transcendante ». Pour cette fusion en Dieu, au terme de cette ténèbre, une perte nécessaire, non seulement du moi, mais de l'acte même d'aimer: «Une fois, je voyais Dieu en si grande clarté, beauté et plénitude, que jamais je ne l'avais vu en si grande clarté ni en ce mode si plein. Et là je ne voyais pas l'amour, et alors je perdis l'amour que je portais, et je devins non-amour ». Énoncé de scandale, qui dit en un même souffle la rencontre du dieu et l'abolition du principe même qui le fonde. Mais ceci, encore, qui s'enchaîne sur ce «non-amour ", comme sa condition : « Dans l'immense ténèbre, je vois la Trinité sainte, et dans la Trinité, aperçue dans la nuit, je me vois moi-même, debout, au centre ${ }^{5}$ ». Angèle, femme advenue comme sujet, après qu'elle soit dénuée d'amour. Et ce n'est pas un hasard si C.-A. Bernard, attentif à la qualité "sensible» de la "perception mystique", en appelle à d'autres femmes vouées à spiritualité - Angèle, à nouveau, Julienne de Norwich, Hildegarde de Bingen, leurs prophéties, visions, ravissements. Vaste « imaginaire mystique » donateur de sens, ainsi qu'il en ira avec Jean de la Croix. Mais des visions à proprement parler à vide. Angèle : « je ne vois rien, qui ait forme, et cependant alors je vois tout et je ne vois rien». Dépassement de l'image, irruption de l'«informel». Toute mystique, rappelle l'A., est refus de forme, de concept, d'image. Denys l'Aréopagite en avait aux premiers temps posé l'absolu principe. Mais il est ici du plus grand intérêt, pour une épistémologie bien 
comprise de la mystique, que cette obligation d'abstraction soit signifiée au cœur de l'analyse de la voie « sensible » vers Dieu.

S'il n'est pas, d'un âge à l'autre, de " progression » dans la conformité à Dieu, sa volonté, son fils, il est cependant des insistances singulières. La folie de la croix, la «folie de l'amour du Père ", s'entendent dans l'observance de l'histoire évangélique comme source de sens : n'entre pas en communion de folie qui veut - il y faut discipline, itinéraire, exercice. Eckhart, Suso, Ignace, lient cette communion à une exacte herméneutique du Livre. Alors on peut, attestant la "proximité de Dieu et de l'homme", aller jusqu'à magnifier du Christ le "cœur blessé »: Gertrude d'Helfta, Marguerite-Marie Alacoque, Jean Eudes, procèdent de cette spiritualité aux frontières mouvantes d'une entreprise dévotionnelle, et d'une offrande mystique. Le temps vient alors où théologie et mystique, sans entrer pour autant en divergence ni opposition, se découplent. Bérulle, de Condren, Olier, se situent dans l'espace de cette disjonction. Il est certes pour eux, selon la formule de l'A., une "correspondance étroite entre les modes d'union à Dieu, et ceux qui regardent les opérations de l'âme ». Mais à la «transformation divinisante » de ce modeci, Bérulle privilégie une perspective " proprement religieuse ", fondée sur le sacerdoce, la prière d'humilité, l'explosion charnelle de la grâce. Ainsi peut-on lire L'élévation sur sainte Madeleine $e^{6}$ : une somptueuse écriture de la conformation au Christ, où vie et mort, amour et désir, oscillent en permanence entre symbolique et signature du réel. Qu'est-ce alors que le Christ ?, demande l'A. : «un ordre singulier du réel », qui sollicite un lien de pleine «servitude». Et ce «réel» se dit en toute netteté, loin d'un silence désiré par d'autres voies mystiques. «Intelligibilité positive ", écrit C.-A. Bernard. Mais on voit que le chemin est court entre exigence de "concrétude» et abandon sacrificiel: cette soumission d'âme et de corps s'accomplit le plus exactement en 1 'expérience létale de Thérèse de Jésus. Non le Christ, mais l'homme Jésus - retour à l'ordre d'un avènement d'histoire, dont d'autres voies mystiques avaient tenté de s'affranchir. L'ouvrage de C.-A. Bernard est l'exposé, en excellence, des voies d'une spiritualité à raison du monde et de sa passion.

21 Bérulle, en cette conformation au Christ, est ici présent en ce monde et cette passion. Présent parmi d'autres, fut-il impair parmi ses pairs. Mais il est acteur central et décisif en l'ouvrage d'Yves Krumenacker - central, par l'implosion même de ses héritages.

Il en va des phénomènes opérant dans le territoire de la spiritualité ou de la mystique, comme il en est des mouvements culturels: leur qualification est affaire de contemporains hostiles, ou de disciples d'après-coup, tenant là occasion de légitimité par procuration. On sait que nul grand énonciateur du pur amour ne s'est qualifié de quiétiste, et que la nomination fut le fait des adversaires de cette doctrine d'oblation parfaite. Ainsi de ce que l'on appelle l'école «française » de spiritualité dans la première moitié du XVII ${ }^{e}$ siècle. Il faut attendre $\mathrm{H}$. Brémond pour que cette expression fasse effet de connaissance : par elle pouvaient entrer en consonance des vocations spirituelles, des allégeances mystiques, autour de quelques principes relativement consensuels pour faire sens et repères dans une histoire heurtée, traversée de mille repentirs. L'entreprise de mise au net conduite par l'A. ne laisse que peu de place, et de crédit, au partage des eaux proposé par Brémond en 1921. Si bien que le titre de l'ouvrage peut paraître en partie trompeur, quand il a pour fin de proposer une lecture infiniment plus souple, et diverse, des expériences spirituelles au siècle des saints.

Cet ouvrage est de la main d'un seul, mais fruit d'une réflexion collective au long cours conduite, dans le cadre de la faculté de théologie de Lyon, par différents représentants 
des cercles religieux se réclamant de l'héritage de Bérulle. Parler d'école "française ", c'est avant tout parler d'un argumentaire bérullien traversant différentes assignations ecclésiales, voire doctrinales, et y autorisant des singularités remarquables. Oratoriens, bien sûr, dont Bérulle fonda la congrégation en France, carmélites, dont il favorisa l'implantation, mais aussi bien lazaristes, sulpiciens avec Olier, eudistes, etc. Mais à trop vouloir partager l'héritage, il advient que le capital se disperse, et perde, dans la multiplicité des réseaux qui le sollicitent, sa fécondité originelle. Au demeurant, l'A. remarque que le bérullisme constitue d'emblée moins un héritage bien configuré, qu'une aventure singulière, propre à un homme d'excellence engagé dans un siècle de pleines tourmentes : Réforme et Ligue, Église en décadence, affluences mystiques, engagements missionnaires - une somme exceptionnelle de défis et de luttes où les incertitudes de la foi répondent aux blessures du monde. Bérulle est comptable de ce temps et de ces faillites. Aussi bien sa doctrine connait-elle des inflexions majeures, qui en interdisent un énoncé monotone. De son "Bref discours de 1 'abnégation intérieure" ( 1597) à son "Discours de l'état et des grandeurs de Jésus » (1623), l'A. montre avec précision le passage d'une spiritualité essentialiste, dans le droit-fil de la mystique rhénane, à une ferveur christocentrique, qui pose qu'on ne peut atteindre Dieu que par un «fait historique ", l'incarnation de ce dieu en cet homme. On peut, bien entendu, rappeler que dans le cercle de haute spiritualité qui se forme très tôt autour de Barbe Acarie, la mystique abstraite de Benoît de Canfield, par exemple, pour s'en tenir à son vecteur le plus irradiant, n'ignore pas la nécessaire mémoire et « imitation de Jésus ». Et que tout au long de son itinéraire doctrinal, jamais Bérulle, au cœur même de la spiritualité fondée sur l'humanité de Jésus, ne cesse de proclamer le néant de la créature, seul capable d'être habité de Dieu. On le voit : l'« école française «de spiritualité n'est ni école - il eut fallu un argumentaire unifié, une volonté de diffusion organisée, l'un et l'autre ici manquants ni, à rigoureusement parler, "française ", puisque pour une large part héritière des grands textes mystiques venus de Flandres, d'Espagne ou d'Italie. Et c'est bien là l'intérêt majeur de l'ouvrage : en finir avec une fiction historique.

De la mystique de l'essence à la «métaphysique de la relation » à Dieu par la figure du Christ-homme, il est clair en effet que le bérullisme ne pouvait valoir référence stable, s'inscrivant en des espaces de sens de trop vaste amplitude. Si donc plus d'un spirituel du XVIIe, mais plus encore aux siècles suivants - car Bérulle disparut très vite et très curieusement du paysage de la théologie mystique -, s'en réclame, c'est d'avoir, chacun pour sa propre économie de pensée, prélevé dans l'héritage lumineux du cardinal les leçons appropriées. Bourgoing, gardien de la doctrine, Gibieuf, Vincent de Paul, Olier, H. M. Boudon, Eudes, Louise de Marillac, de Condren, privilégient des raisons singulières : néantisation et humilité, absolu de l'essence divine, homme-dieu étranger au monde, Dieu présent au contraire en ce monde même, corruption de la créature, Dieu trinitaire, dévotion à l'enfance du Christ, etc. Et l'argument propre à la théologie négative, qui depuis des siècles rappelle la leçon maîtresse de Denys l'Aréopagite. Le bérullisme, cette "spiritualité complexe ", n'en finit pas de se transformer au fil des générations, et son influence de se diluer autour de quelques raisons communes. Un " ton commun ", dit l'A. : union intime à la vie de Jésus, pratique ardente de l'oraison, etc. - et l'on suit avec intérêt le cheminement des thèses $d u$ " maître » en ceux qui s'en réclament. Notices brèves, sans doute, courtes biographies spirituelles, où l'on saisit la distension d'un corps déjà mobile de pensers, système de transformations plus que référentiel stabilisé. 
Mais au-delà de cette profusion de spiritualités habitant les interstices mêmes du bérullisme, l'A. marque avec justesse l'affleurement progressif, en ces décennies d'« invasion mystique ", du thème de l'homme intérieur, et de la catégorie du sujet qui en consacre la modernité. Peut-être doit-on voir en cette neuve insistance portée sur le "fond de l'âme » - appellation rhénane -, ou la "pointe de l'esprit» - dénomination salésienne -, l'une des raisons de la dispersion de la doctrine de Bérulle en mille orients. De cette thématique viendra en fin de siècle la quête du pur amour, au terme d'une histoire emplie de conflits et d'anathèmes, qui sont précisément au fondement de toute «école ». S'il n'est pas de spiritualité « française », il est une problématique d'amour pur qui traverse l'ensemble des énoncés mystiques du XVII ${ }^{e}$. Si bien que, selon des modalités qu'il serait passionnant de préciser, une intrigue ne cesse de se nouer entre la règle bérullienne de la "contemplation du verbe incarné ", l'imitation "d'un homme-dieu pouvant aller jusqu'à diviniser l'homme", selon l'expression de l'A., - et le lent engendrement de la formule du pur amour, depuis François de Sales jusqu'à Fénelon. Au milieu du siècle, un homme donne à cette formule son éclat et sa rigueur exacte, le dominicain Alexandre Piny ${ }^{7}$. C'est à peu près à ce moment que le visage de Bérulle disparaît de la scène mystique. S'il revient aujourd'hui en l'un des premiers rangs, ainsi que le démontre l'A., c'est peut-être parce que cette « divinisation de l'homme » n'était chez le cardinal que l'autre nom du sujet venu à son accomplissement, et dont l'édit de pur amour porte la passion à sa plus forte exigence. S'il n'est pas d'école française de spiritualité, c'est parce que celui qui aurait pu en être le maitre a fait rencontre, combien brutale, d'un défi plus ardent qu'hérésies de son temps, ou disputes théologiennes: l'impératif d'assumer d'un seul coup la pure violence au cœur de l'amour même, qui retourne aussitôt le monde comme un gant.

Les multiples figures présentées en ces ouvrages, soit d'évidence féminines, soit porteuses de féminité ardente, forment cortège trop insistant pour qu'elles ne finissent pas par dessiner un système cohérent, contraignant, qui travaille en son cœur l'ensemble des territoires de mystique. Sans doute la leçon de spiritualité ne saurait-elle s'en trouver aussitôt, et entièrement, mise au net : tant d'autres instances - proprement théologiques, ecclésiales, doctrinales, historiques, sociales - œuvrent à la mise en forme de la spiritualité occidentale. Mais on peut être assuré qu'au travers même de ces instances, si l'on advient à leur ultime raison, rencontrer Dieu c'est, à coup sûr, faire rencontre du féminin comme principe d'engendrement de la formule, et de la parole, mystiques.

\section{NOTES}

1. Pour mémoire: Une inconnue célèbre. Madeleine Lebouc/Pauline Lair Lamotte (1853-1918), Paris, Anthropos, 1993 (cf. Arch. $\mathrm{n}^{\circ}$ 84.61) ; Les stigmates de l'hystérique et la Peau de son évêque. Laurentine Billoquet (1862-1936), Paris, Anthropos, 1993 (cf. Arch. $\mathrm{n}^{\circ}$ 84.62) ; L'Autobiographie d'un paranoïaque. L'abbé Berry (1878-1947) et le roman de Billy, «Introibo », Paris, Anthropos, 1994 (cf. Arch. n 88.55) et L'Orpheline de la Bérésina. Thérèse de Lisieux (1873-1897). Essai de psychanalyse sociohistorique, Paris, Cerf, $19 \%$ (cf. Arch. n 96, pp. 41-50). Cet ensemble de "monographies " trouve sa complétude avec Mystique et féminité, Paris, Cerf, 1997. Sur ce dernier ouvrage, cf. D. vIDAL, «Le défi des 
interprétations: figures de femmes et expérience mystique ", Archives de Sciences Sociales des Religions, 102 (avril-juin), 1998, pp. 29-33.

2. Cf. notamment Caroline W. BYNUM, Fragmentation and redemption: Essays on Gender and the Human Body in Medieval Women, Berkeley, University of California Press, 1991 ; Holy Feast and Holy Fast: The religious Significance of Food to Medieval Women, ibid., 1987.

3. Sur ces deux grandes figures, cf. D. VIDAL, Critique de la raison mystique. Benoit de Canfield, possession et dépossession au XVII ${ }^{e}$ siècle, Grenoble, J. Millon, 1990 et Le coup terrible du néant, introduction à la réédition de La Perle évangélique, Grenoble, Jérôme Millon, 1997 (cf. Arch. 102, n - 126).

4. C.-A. BERNARD, Le Dieu des mystiques ; les voies de l'intériorité, Paris, Cerf, 1994.

5. C.-A. BERNARD utilise une traduction plus « apaisée » : «En cette Trinité (...) je suis et repose en son milieu... Il me semble que l'essentiel, ici, est moins ce repos, que ce je “suis" ».

6. Je me réfère à la dernière édition de l'ouvrage de Pierre Bérulle, présentée par Joseph Beaune, Grenoble, J. Millon, 1998.

7. Cf. Daniel VIDAL, L'Édit de pur amour, suivi des Lettres spirituelles d'A. Piny (1683-1686), J. Millon, 2000 (à paraître).

\section{AUTEUR}

\section{DANIEL VIDAL}

Centre d'Études des Mouvements Sociaux, CNRS-EHESS 